\title{
Is the game over for PD-1 inhibitors in EGFR mutant non-small cell lung cancer?
}

\author{
Maria A. Velez ${ }^{1}$, Timothy F. Burns ${ }^{2,3}$ \\ ${ }^{1}$ Department of Internal Medicine, University of Pittsburgh Medical Center, Pittsburgh, PA, USA; ${ }^{2}$ Department of Pharmacology and Chemical \\ Biology, University of Pittsburgh, Pittsburgh, PA, USA; ${ }^{3}$ Department of Medicine, Division of Hematology-Oncology, UPMC Hillman Cancer \\ Center, Pittsburgh, PA, USA \\ Correspondence to: Timothy F. Burns, MD, PhD. UPMC Hillman Cancer Center, Hillman Cancer Center Research Pavilion, Office: Suite 2.18e Lab: \\ 2.7, 5117 Centre Avenue, Pittsburgh, PA 15213-1863, USA. Email: burnstf@upmc.edu. \\ Provenance: This is an invited article commissioned by the Section Editor Hengrui Liang (Department of Thoracic Surgery, Guangzhou Medical \\ University, Guangzhou, China). \\ Comment on: Lisberg A, Cummings A, Goldman JW, et al. A Phase II Study of Pembrolizumab in EGFR-Mutant, PD-L1+, Tyrosine Kinase Inhibitor \\ Naïve Patients With Advanced NSCLC. J Thorac Oncol 2018;13:1138-45.
}

Submitted Apr 05, 2019. Accepted for publication Apr 18, 2019.

doi: $10.21037 /$ tlcr.2019.04.09

View this article at: http://dx.doi.org/10.21037/tlcr.2019.04.09

The immune checkpoint inhibitor pembrolizumab, has been shown to be efficacious and to have significant and durable antitumor activity in a subset of patients with advanced nonsmall cell lung cancer (NSCLC). Most notably in NSCLC with high expression of the programmed death ligand-1 (PD-L1). Similarly, there is abundant data to support the use of epithelial growth factor receptor (EGFR) tyrosine kinase inhibitors (TKIs) as first-line therapy in patients whose tumors harbor EGFR mutations (1). However, these patients will inevitably progress on these targeted agents and there has been strong interest in examining whether immune checkpoint inhibitors could be effective in these patients (2). To date, the data for use of immune checkpoint inhibitors either after EGFR TKIs or in combination with EGFR TKIs has been extremely disappointing (3). Data to support the use of these agents in the front-line setting in EGFR mutant PD-L1 positive NSCLC patients, was encouraging but sparse. While there is ample published data showing that there are associations between PDL1 expression and EGFR signaling in NSCLC, whether this could affect response to immunotherapy is not well established and the use PD-1 and PD-L1 inhibitors in this clinical setting remains controversial (4). In a recent study, Lisberg et al. examined whether the anti-PD-1 agent, pembrolizumab could be effective in EGFR mutant patients prior to receiving an EGFR TKI (5).
Preclinical data to support or reject the use of PD-1 inhibitors such as pembrolizumab in patients with EGFR mutant NSCLC is conflicting. It is well established that patients who harbor EGFR mutations have lower nonsynonymous mutational burden than EGFR WT patients. This is important as the tumor mutational burden has been associated with higher response to PD-1/PDL1 inhibitors (6). Similarly, data from pre-clinical studies show that EGFR-mutated NSCLC tumors lack T-cell infiltration and have a more immunosuppressive tumor microenvironment, which could potentially negatively impact the response to PD-1/PD-L1 inhibitors (7). Conversely, there is also data to suggest that activating EGFR mutations led to PD-L1 upregulation in NSCLC as well as animal models showing increase survival after PD-1 therapy in EGFR driven adenocarcinoma (8-10). However, similar efficacy has not been observed in the clinic. A retrospective study compared the objective response rate (ORR) after treatment with PD-1/PD-L1 inhibitors in EGFR WT patients and EGFR mutant patients (11). Of the 22 evaluable patients with $E G F R$ mutations, most had already progressed on an EGFR-TKI before receiving treatment with a PD-1/PD-L1 inhibitor, and three received combination therapy with both agents after progression. Only 1 out of the 22 patients in the EGFR mutant group obtained an objective response to treatment compared to 7 
out of the 30 patients with EGFR WT tumors, suggesting that $E G F R$ mutant patients do not respond to anti-PD-1/ PD-L1 inhibitors as well as EGFR WT patients do. Furthermore, a pooled analysis which included data from five clinical trials evaluating PD-1/PD-L1 inhibitors in NSCLC patients, showed that prolonged overall survival (OS) was only observed in EGFR WT patients but not the EGFR mutant patients (12).

In contrast to the preclinical and clinical data in EGFR mutant patients who received immunotherapy after an EGFR TKI, experience from the KEYNOTE-001 trial suggested that patients with EGFR mutant tumors may have improved survival outcomes after receiving first-line pembrolizumab (13). In the KEYNOTE-001 trial, a small number of EGFR TKI naïve EGFR mutant patients $(\mathrm{n}=4)$ received pembrolizumab and had an improved ORR, progression-free survival (PFS) and OS after treatment with pembrolizumab compared to EGFR mutant patients who had received previous treatment with an EGFR TKI and subsequently received pembrolizumab (ORR of $50 \%$ and median OS of 18 months compared to ORR of $4 \%$ and OS of 4 months, respectively) (13). To formally test whether pembrolizumab could be effective in the EGFR TKI naïve setting, Lisberg et al. conducted a phase II trial to evaluate the response of EGFR mutant, PD-L1 positive $(\geq 1 \%)$, TKI naïve patients with advanced NSCLC, to the PD-1 inhibitor, pembrolizumab (5). The inclusion criteria comprised the following; patients with advanced NSCLC, with sensitizing or non-sensitizing EGFR mutations who had PD-L1 positive tumors, defined as $\geq 1 \%$ tumor membranous staining by immunohistochemistry (IHC) using the $22 \mathrm{C} 3$ pharmDx test. This study excluded patients who had previously received treatment with either an EGFR TKI or a PD-1/PD-L1 inhibitor. The primary endpoint was ORR to pembrolizumab using the Response Evaluation Criteria in Solid Tumors 1.1, the secondary endpoints included safety of the drug as well as PFS and OS.

Of the 25 patients that were screened for the study, 14 patients $(56 \%)$ screened failed and only 11 patients were enrolled. Most of these patients were treatment naïve (82\%), had PD-L1 expression levels $\geq 50 \%$ (73\%), were never smokers $(54 \%)$ and were female $(63 \%)$. The duration of follow-up was 7.7 months (233 days). Efficacy results showed that the only patient who had an objective response (ORR of 9\%) to pembrolizumab at the time of data cut-off, did not truly harbor an EGFR mutation, and this was the result of a laboratory error. Taking this into consideration the ORR was $0 \%$ for the 10 patients who met inclusion criteria. In terms of adverse events (AE), 5 patients (46\%) experienced treatment related $\mathrm{AE}$, one of which had grade 3 transaminitis. Based on these results the study was discontinued due to futility.

After the study was discontinued, 9 patients underwent subsequent therapy. Two patients received chemoradiation and the remaining 7 patients received erlotinib for a median duration of treatment of about 3.5 months. Two patients died on erlotinib and 6 out of 7 patients who received TKIs experienced side effects. Notably, one patient experienced grade 5 pneumonitis thought to be secondary to the EGFR TKI although a causal link to prior pembrolizumab could not be excluded.

The aims of this study were to evaluate the feasibility and safety of pembrolizumab in patients with PD-L1 positive, EGFR mutant NSCLC before an EGFR TKI. This study hypothesized that the use of pembrolizumab in this patient population, before EGFR TKIs would lead to better clinical outcomes, defined as an ORR greater than $26 \%$. Three key components would warrant the use of pembrolizumab in this setting: (I) the clinical benefit of pembrolizumab would have to be greater before administration of an EGFR TKI; (II) the benefits with an EGFR TKI would be unaffected by prior therapy; and (III) the safety profile would be equivalent as if the agents were given in reversed. The present study failed to demonstrate a clinical benefit from pembrolizumab in EGFR mutant patients and potentially raised questions about subsequent side effects with EGFR TKIs given as the next therapy.

As authors allude to in the manuscript, this study has several limitations, an important one is the small sample size of the study and the fact that it enrolled less than half of the patients that it initially sought out to do. This decreases the power of the study, and thus its ability to truly evaluate the feasibility of a therapeutic intervention in a population. Another important limitation of the study is the short follow-up time of only about 7 months to data cut-off, whereas studies showing the clinical benefits of pembrolizumab in the de novo setting had a follow-up of about twice as long (14). Furthermore, most of the patients included in the study were female $(63 \%)$ and never smokers (54\%), two populations that seem to derive less benefit from the PD-1/PD-L1 inhibitors $(13,15)$. Conversely, most of the patients were also treatment naïve (82\%) and PD-L1 $\geq 50 \%(73 \%)$ which are associated with improved response to pembrolizumab (16).

Certainly, while the study has the limitations mentioned 
above, it is important to note its strengths. This is the first prospective trial to evaluate the use of $\mathrm{PD}-1$ inhibitors in the first-line setting for EGFR mutant patients. Before the publication of this study all the data to evaluate this treatment strategy in the EGFR mutant population had been speculative, coming from retrospective studies and subset analysis from clinical trials designed to answer different questions. Furthermore, while sample size of the study was small it is representative of the patient population affected by EGFR mutant NSCLC (1). Additionally, the results from the study support previously published data indicating the lack of benefit from PD-1/PD-L1 inhibitors in EGFR mutant NSCLC as authors conclude that pembrolizumab is not an appropriate therapeutic choice in patients with treatment naïve EGFR mutant, PD-L1 positive NSCLC.

Although single agent immunotherapy is unlikely to be effective in EGFR mutant patients, other trials have assessed the combination of anti-PD-1/anti-PD-L1 agents in combination with chemotherapy or EGFR TKIs in the EGFR mutant population, with mixed results. The IMpower 150 trial evaluated the combination of carboplatin, paclitaxel and bevacizumab plus or minus the PD-L1 inhibitor, atezolizumab, in patients with both EGFR WT and EGFR mutant NSCLC (17). Results showed that PFS was significantly longer in EGFR mutant patients who received atezolizumab in addition to chemotherapy and bevacizumab as compared to those who only received chemotherapy and bevacizumab (9.7 vs. 6.1 months, HR $0.59 ; 95 \%$ CI, 0.37 to 0.94$)$. It is important to denote that there were 108 patients in this subgroup of the study, but this also included patients with $A L K$ translocations. Additionally, most of the patients with $E G F R$ mutations included in this study had already received and failed first line treatment with EGFR TKIs. Thus, these results cannot be extrapolated to the treatment naïve setting in EGFR mutant patients. Similarly, the PACIFIC trial compared durvalumab, a PD-L1 inhibitor, as consolidation therapy after chemotherapy in patients with stage III NSCLC. In a subgroup analysis, patients with EGFR mutations also benefited from durvalumab after chemotherapy. However, this failed to reach statistical significance and like the aforementioned study these patients received fist-line treatment with another agent before receiving durvalumab (18). The TATTON trial was a phase Ib study that evaluated the combination of osimertinib in combination with durvalumab in both the EGFR TKI pretreated patients as well as in EGFR TKI treatment-naïve patients. Unfortunately, the rates of treatment-related $\mathrm{AE}$ were high and $38 \%$ of patients developed interstitial lung disease, leading to study discontinuation (19). Similarly other published studies that have evaluated the combination or EFGR TKIs with PD-1 or PD-L1 inhibitors have been limited by the toxicity rates, leading in many instances to treatment discontinuation (20).

To our knowledge, this phase II trial conducted by Lisberg et al. is the only prospective published study thus far that has evaluated PD-1 inhibitors as monotherapy in the front-line setting for patients with EGFR mutant NSCLC. While PD-L1 identification by IHC seems to be a good prognostic and predictive indicator to response in NSCLC, this does not seem to be the case for EGFR mutant patients. As thus, with the published data that is currently available, there is not a current role for the use of PD-1/PDL-1 inhibitor in the front line setting for EGFR mutant patients. After progression of an EGFR TKI, EGFR mutant patients have traditionally been treated with a platinum doublet and recent data suggest that these patients may benefit from combined chemoimmunotherapy. In addition, with development of new immunotherapeutic agents as well as possibly other predictive biomarkers, this is likely not the end for the use of immunotherapy in EGFR mutant patients.

\section{Acknowledgments}

None.

\section{Footnote}

Conflicts of Interest: The authors have no conflicts of interest to declare.

\section{References}

1. Sharma SV, Bell DW, Settleman J, et al. Epidermal growth factor receptor mutations in lung cancer. Nat Rev Cancer 2007;7:169-81.

2. Kim HJ, Choi EY, Jin HJ, et al. Relationship between EGFR mutations and clinicopathological features of lung adenocarcinomas diagnosed via small biopsies. Anticancer Res 2014;34:3189-95.

3. Liang H, Liu X, Wang M. Immunotherapy combined with epidermal growth factor receptor-tyrosine kinase inhibitors in non-small-cell lung cancer treatment. Onco Targets Ther 2018;11:6189-96.

4. Zhang $\mathrm{N}$, Zeng $\mathrm{Y}, \mathrm{Du} \mathrm{W}$, et al. The EGFR pathway is involved in the regulation of PD-L1 expression via the 
IL-6/JAK/STAT3 signaling pathway in EGFR-mutated non-small cell lung cancer. Int J Oncol 2016;49:1360-8.

5. Lisberg A, Cummings A, Goldman JW, et al. A Phase II Study of Pembrolizumab in EGFR-Mutant, PD-L1+, Tyrosine Kinase Inhibitor Naïve Patients With Advanced NSCLC. J Thorac Oncol 2018;13:1138-45.

6. Rizvi H, Sanchez-Vega F, La K, et al. Molecular Determinants of Response to Anti-Programmed Cell Death (PD)-1 and Anti-Programmed Death-Ligand 1 (PD-L1) Blockade in Patients With Non-Small-Cell Lung Cancer Profiled With Targeted Next-Generation Sequencing. J Clin Oncol 2018;36:633-41.

7. Dong ZY, Wu SP, Liao RQ, et al. Potential biomarker for checkpoint blockade immunotherapy and treatment strategy. Tumour Biol 2016;37:4251-61.

8. Akbay EA, Koyama S, Carretero J, et al. Activation of the PD-1 pathway contributes to immune escape in EGFRdriven lung tumors. Cancer Discov 2013;3:1355-63.

9. Chen N, Fang W, Zhan J, et al. Upregulation of PDL1 by EGFR Activation Mediates the Immune Escape in EGFR-Driven NSCLC: Implication for Optional Immune Targeted Therapy for NSCLC Patients with EGFR Mutation. J Thorac Oncol 2015;10:910-23.

10. Azuma K, Ota K, Kawahara A, et al. Association of PDL1 overexpression with activating EGFR mutations in surgically resected nonsmall-cell lung cancer. Ann Oncol 2014;25:1935-40.

11. Gainor JF, Shaw AT, Sequist LV, et al. EGFR Mutations and ALK Rearrangements Are Associated with Low Response Rates to PD-1 Pathway Blockade in Non-Small Cell Lung Cancer: A Retrospective Analysis. Clin Cancer Res 2016;22:4585-93.

12. Lee CK, Man J, Lord S, et al. Clinical and Molecular

Cite this article as: Velez MA, Burns TF. Is the game over for PD-1 inhibitors in EGFR mutant non-small cell lung cancer? Transl Lung Cancer Res 2019;8(Suppl 4):S339-S342. doi: 10.21037/tlcr.2019.04.09
Characteristics Associated With Survival Among Patients Treated With Checkpoint Inhibitors for Advanced NonSmall Cell Lung Carcinoma: A Systematic Review and Meta-analysis. JAMA Oncol 2018;4:210-6.

13. Rizvi NA, Hellmann MD, Snyder A, et al. Cancer immunology. Mutational landscape determines sensitivity to PD-1 blockade in non-small cell lung cancer. Science 2015;348:124-8.

14. Reck M, Rodríguez-Abreu D, Robinson AG, et al. Pembrolizumab versus Chemotherapy for PD-L1Positive Non-Small-Cell Lung Cancer. N Engl J Med 2016;375:1823-33

15. Conforti F, Pala L, Bagnardi V, et al. Cancer immunotherapy efficacy and patients' sex: a systematic review and meta-analysis. Lancet Oncol 2018;19:737-46.

16. Garon EB, Rizvi NA, Hui R, et al. Pembrolizumab for the treatment of non-small-cell lung cancer. N Engl J Med 2015;372:2018-28.

17. Socinski MA, Jotte RM, Cappuzzo F, et al. Atezolizumab for First-Line Treatment of Metastatic Nonsquamous NSCLC. N Engl J Med 2018;378:2288-301.

18. Antonia SJ, Villegas A, Daniel D, et al. Durvalumab after Chemoradiotherapy in Stage III Non-Small-Cell Lung Cancer. N Engl J Med 2017;377:1919-29.

19. Ahn MJ, Yang J, Yu H, et al. 136O: Osimertinib combined with durvalumab in EGFR-mutant non-small cell lung cancer: Results from the TATTON phase Ib trial. J Thorac Oncol 2016;11:S57-166.

20. Soo RA, Lim SM, Syn NL, et al. Immune checkpoint inhibitors in epidermal growth factor receptor mutant non-small cell lung cancer: Current controversies and future directions. Lung Cancer 2018;115:12-20. 